\title{
An EFTEM Study of Polyimide Adhesion to Single-Walled Carbon Nanotube Bundles
}

\author{
N.D. Evans, ${ }^{*}$ C. Park,** R. Crooks, ${ }^{* * *}$ E.J. Siochi, ${ }^{* * * *}$ and E.A. Kenik* \\ *Oak Ridge National Laboratory, PO Box 2008, Oak Ridge, TN 37831-6376 \\ ** ICASE, ***Lockheed Martin Space Operations, and ****Advanced Materials and Processing \\ Branch, NASA Langley Research Center, Hampton VA 23681-2199
}

When reinforced with single-walled carbon nanotubes (SWNT), significant benefits in strength and electrical properties of polyimide are expected. However, uniform dispersion of SWNT throughout the polymer matrix may be compromised due to the lack of adhesion between the polymer and the SWNT.[1] For uniform dispersion of SWNT in the polymer matrix to occur, the solvated polymer must wet the non-reactive SWNT surface during production. In this study, energy-filtered transmission electron microscopy (EFTEM) has been used to reveal that polyimide has adhered to prepared SWNT surfaces within an effectively reinforced composite.

Purified laser ablated SWNT, having diameters $\sim 1.4 \mathrm{~nm}$, were combined with polyimide $\left(\mathrm{C}_{35} \mathrm{~N}_{3} \mathrm{O}_{7} \mathrm{H}_{17}\right)$ to produce a series of SWNT-polyimide composite films having a SWNT concentration of $0.1 \mathrm{wt} \%$. Processing details are described elsewhere.[2,3] The TEM specimens were prepared by redissolving the composite film in the solvent used for the synthesis, and dispersing the dilute solution onto a holey carbon film. The EFTEM was performed using a Philips CM30, with a $\mathrm{LaB}_{6}$ cathode and a Gatan Image Filter (GIF). Filtered images were typically obtained with $>100 \mathrm{nA}$ probe current, $\sim 1700-3900 \mathrm{x}$ TEM magnification, 2.9 mrad incident beam divergence $\alpha, 4.8 \mathrm{mrad}$ collection half angle $\beta, 30 \mathrm{eV}$ filter slit width, and $10-15 \mathrm{~s}$ exposures. The large probe current and slit width were required to obtain sufficient energy-filtered signal from these thin specimens.

Figure 1 shows a zero-loss image of the redissolved SWNT-polyimide nanocomposite. The SWNT bundles were seen to be both across holes and on the carbon substrate. Beads of material were observed which often appeared to encompass and wick the bundle. Both oxygen and nitrogen elemental maps (Fig. 2a, and 2b) indicate the beads are polyimide. Pixel intensity profiles, obtained across the bundles at regions $\mathrm{A}, \mathrm{B}$, and $\mathrm{C}$ within the oxygen map are presented in Figure $3 \mathrm{a}$. Oxygen, presumed to be from the polyimide coating, was consistently detected along bundles, indicating good adhesion of the polyimide to the SWNT. Planned experiments to examine oxygen near-edge structures may help distinguish covalently bonded oxygen associated with polyimide, from adsorbed oxygen on the bundles.

Interestingly, the nitrogen mapping was problematic due to beam interactions with the SWNT bundles. Image drift correction across the entire image (Fig. 2b) was imperfect due to slight translations and rotations to those bundles lying across holes. However, bundles on the carbon substrate did not suffer this effect as much. (This may be due to the carbon substrate acting as a local heat sink or by adding structural stability to the bundle.) It should be noted that the oxygen component images, acquired prior to the nitrogen component images, did not suffer from this problem. Intensity profiles (Fig. 3b) from regions $\mathrm{D}$ and $\mathrm{E}$ in the nitrogen elemental map may reliably be used to mark the nitrogen associated with the polyimide. However, an acid treatment performed during SWNT production may be contributing to this signal.[4] 


\section{References}

[1] Kevin D. Ausman et al., J. Phys. Chem, 104 (2000) 8911

[2] J. Simpson, Z. Ounaies, and C. Fay, Mater. Res. Soc. Symp. Proc. 459 (1997) 59.

[3] C. Park, R. Crooks, E.J. Siochi, N.D. Evans, and E.A. Kenik, Chem. Phys. Lett, submitted

[4] Research through the Oak Ridge National Laboratory SHaRE User Program was sponsored by the Division of Materials Sciences and Engineering, U.S. Department of Energy, under contract DE-AC05-00OR22725 with UT-Battelle, LLC. The authors are grateful for discussions with J. Bentley at ORNL and J.S. Harrison, at NASA Langley.

Fig. 1 - Zero-loss image of redissolved SWNTPolyimide nanocomposite

Fig. 2 - a.) Oxygen elemental map, and b.) nitrogen elemental map

Fig. 3 - Intensity profiles across nanocomposites from indicated regions in a.) $\mathrm{O}$ elemental map, and b.) $\mathrm{N}$ elemental shown in Fig. 2 b.
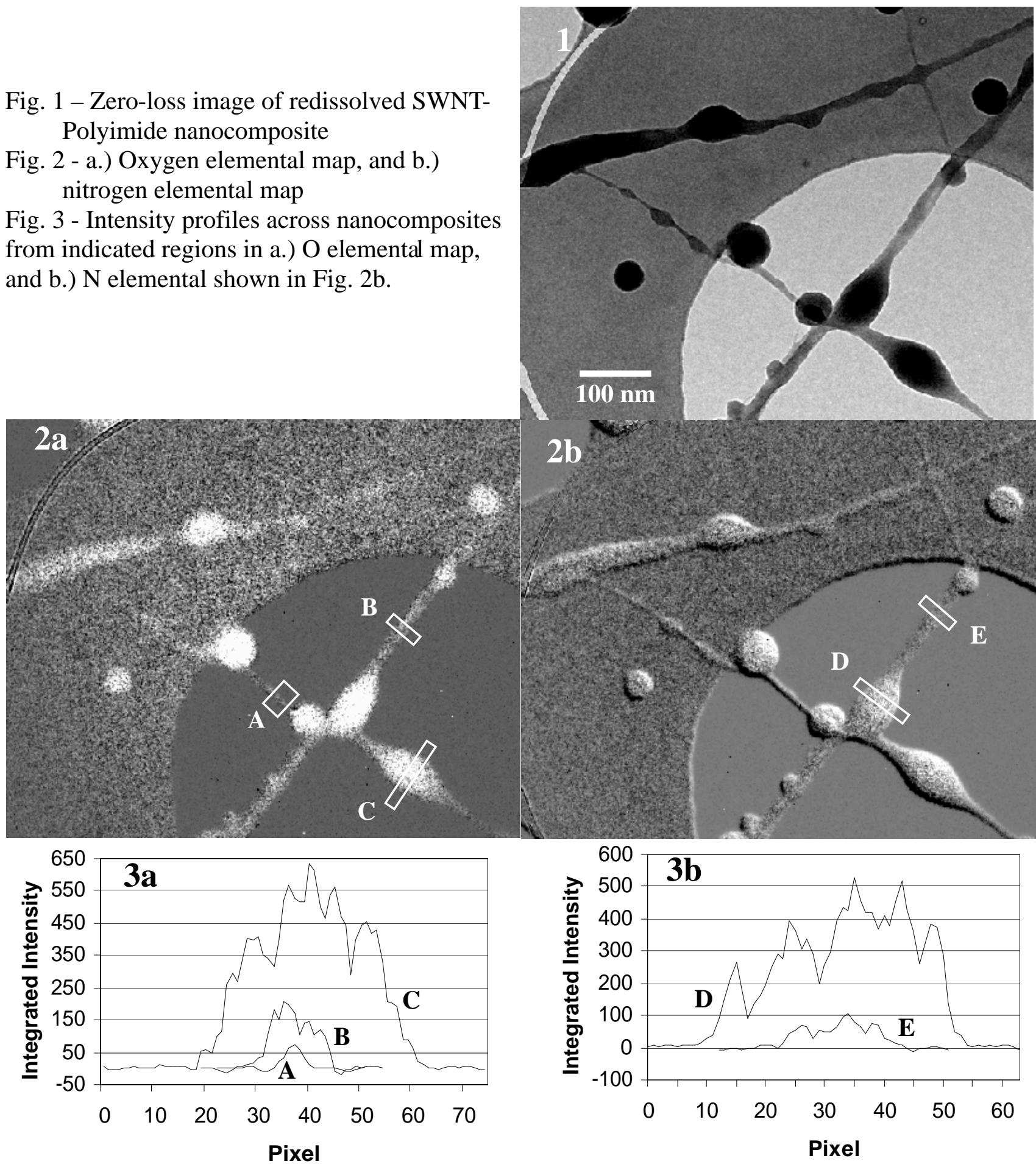